\title{
Study of the Numerical Parameter Optimization Method based on Nano-indentation Process
}

\author{
J.S. Ding, G.Q. Shi, G.F. Shi \\ Changchun University of Science and Technology \\ Ji Lin, China, 130012
}

\begin{abstract}
In order to solve critical process parameters characterize aluminum grating is proposed to optimize the impact of stress calculation values are based on a nano-indentation experiment - Method strain relationship parameters. By establishing numerical approximation model to fit the experimental data of training, and the experimental data for numerical optimization. Experimental results show that: the method to calculate the yield strength aluminum material property parameters, namely strain hardening exponent, and obtained more satisfactory aluminum material properties parameter elastic modulus and yield strength relationship by comparing the value of the deviation can be found in the obvious effectiveness of this approach, but also proved a reliable orthogonal experiment. The research process parameters characterize the relationship between the aluminum grating characterization and constitutive model provides a reference and basis for, and also provide a basis for the development of intelligent raster scored.
\end{abstract}

Keywords-grating aluminum film; substrate; nano-indentation; grating ruling

\section{INTRODUCTION}

Mechanical process issues grating ruling is consistent is the key to troubled grating manufacturing personnel, and long, the film still has not mastered the mechanical properties of the grating and the strain constitutive relation, because the stress grating film scored-strain characterization of the relationship between a aspects dependent on the physical properties of the film material and additional material of the grating itself, more importantly, on the other hand depends on both the equipment and the engraving tool scribe physical geometry. This makes it difficult to extract relations or discriminating between direct and deformation in the experiment. Therefore, to explore suitable for grating fabrication process today, reveal the mechanism of the grating mechanical property characterization constitutive relationship is particularly important characterization methods[1] [2].

US Richardson Grating Laboratory (Richardson Grating Laboratory) after a long period of research and exploration, has been carved out of the world can be directly largest grating, the maximum area of up to $400 \mathrm{~mm} \times 600 \mathrm{~mm}$ [3]. Reed et al., The film separated from the substrate to carry out a conventional tensile test [4], but this test method is particularly difficult to implement, and is generally difficult to obtain the desired tensile curve, and therefore such a method is not easy to obtain a wide range of applications. Shut [5] and Bader [6] Similarly tried separately release film study, just peeling method (thermal stress method) which uses a different, and they have a relatively large film temperature change during the study, so the use of thermal stress the method difficult to obtain mechanical strength of the film was difficult to apply to the film material at room temperature. Shuai Hua Shang et al [7] using the finite element method with the indentation method of combining layers of composite material W/Al bilayer metal film department to do some research to prove that the elastic modulus and hardness of the $\mathrm{W}$ film number the average of the corresponding bulk material parameters varied.

Based on the scoring process of the existing research on the grating is proposed based on indirect indentation experiments, a lot of data, and the data were fitted and gradually approaching to reflect grating constitutive relations approach. Aims to characterize the process of grating material stress - strain constitutive relationship provides the reference and basis, and also provides the basis for the development of intelligent raster scored.

II. THE MECHANISM CHARACTERIZATION OF THE GRATING

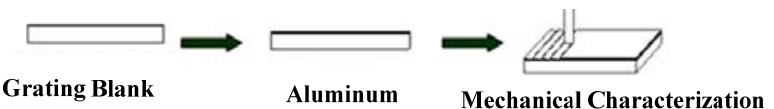

FIGURE I. DEPICTS A SIMPLE FLOW CHART OF MANUFACTURING GRATINGS

As shown in Figure 1, this paper is coated grating blank one or more layers of metal films on a glass substrate and then coated by a diamond knife to perform characterization, in scribing knife through extrusion coating process to make it happen plastic deformation, to form the desired "Groove", i.e., it is a simple process for preparing a ruled grating. Study the fundamental reason is not mastered the mechanical constitutive relation raster layers in the composite aluminum and aluminum grating groove formed under the laws graver understanding of the role is unclear, thus affecting the design and subsequent diamond knife scribe solving process parameters, and even affect the replication slotted grating production quality. Therefore, the mechanical properties of aluminum manufacturing clear grating is particularly important.

\section{GRating Ruling Established the Model Fitting APPROACH}

Based on material mechanics, stress elastoplastic material base member-strain relations power hardening model, 


$$
\sigma=\left\{\begin{array}{cc}
E \varepsilon & \sigma \leq \sigma_{y} \\
R \varepsilon^{n} & \sigma \geq \sigma_{y}
\end{array}\right.
$$

When $\sigma=\sigma y$, there

$$
\sigma_{y}=E \varepsilon_{y}=R \varepsilon^{n}
$$

Wherein the stress, $\varepsilon$ is the strain of the total, $\varepsilon y$ when the stress $\sigma y$ corresponding strain, i.e. the yield strain, $\mathrm{R}$ is the coefficient, and

$$
\varepsilon=\varepsilon_{y}+\varepsilon_{p}
$$

Where, $\varepsilon p$ total strain to remove part of the linear part of the strain can be called plastic strain.

So the load $\mathrm{P}$ can be represented by the following parameters:

$$
P=P\left(h, E, v, E_{i}, v_{i}, \sigma_{y}, n\right)
$$

Wherein, E, v, Ei and vi are compressed material and the modulus of elasticity and Poisson's ratio of the indenter; $\sigma y$ is the yield strength of the pressed material; $\mathrm{n}$ is pressed material strain hardening index. While the above formula can be expressed as:

$$
P=P\left(h, E^{*}, \sigma_{\mathrm{r}}, n\right)
$$

Take $\sigma_{\mathrm{r}}$ and $\mathrm{h}$ as the basic amount, according to the dimension of $\Pi$ theorem, the above equation can be written style,

$$
C=\frac{P}{h^{2}}=\sigma_{\mathrm{r}} \Pi_{1}\left(\frac{E^{*}}{\sigma_{\mathrm{r}}}, n\right)
$$

Thus, the constitutive relation to aluminum expressed as a quadratic curve fitting equation relationship, then it will use nano-indentation measured data for training and approximation, numerical model has been reached for grating ruling process.

\section{NANO-INDENTATION EXPERIMENTS}

\section{A. Sample Preparation}

$79 \mathrm{~g} / \mathrm{mm}$ rough structure of aluminum-chromium film glass substrate layer composite structures were coated by vacuum evaporation method crossing. Aluminized chromium plating film with a thickness of $10 \mu m, 1 \mu m$, respectively, a chrome film is the elastic modulus and Poisson's ratio were 240GPa, 70GPa, 0.3, a glass substrate elastic modulus and Poisson's ratio were 70GPa, 0.3.

\section{B. Test Method}

Swiss CSM's commercial nanoindentation testing research $79 \mathrm{~g} / \mathrm{mm}$ echelle rough, indenter Berkovich indenter for. Indentation depth coverage Echelle mechanical scribing depth of 0.5-5 $\mu \mathrm{m}$, depth interval of $0.5 \mu \mathrm{m}$, the depth of each test was repeated six groups, loaded (Time 10s) by controlling the displacement of the way, Paul load (time 10s), uninstall (time 10 s), a total of 60 group test.

\section{Test Results}

Figure 2 is a resultant indentation load - displacement curve, Table 1 is a set of data indentation. From the figure it can be observed slight curve fluctuations, but the overall trend

\begin{tabular}{|c|c|c|c|c|c|c|c|c|}
\hline $\mathrm{h}_{\max } / \mu \mathrm{m}$ & $\mathrm{K}$ & $\mathrm{x}$ & $\mathrm{H}$ & $\mathrm{E} / \mathrm{GPa}$ & $\mathrm{K}_{\text {mean }}$ & $\mathrm{x}_{\text {mean }}$ & $\begin{array}{l}\mathrm{H}_{\text {mean }} \\
/ \mathrm{GPa}\end{array}$ & $\begin{array}{l}\mathrm{E}_{\text {mean }} \\
/ \mathrm{GPa}\end{array}$ \\
\hline \multirow{6}{*}{0.5} & 8.138 & 1.091 & 0.8634 & 90.4119 & \multirow{6}{*}{13.359} & \multirow{6}{*}{$\begin{array}{l}1.053 \\
2\end{array}$} & \multirow{6}{*}{0.856} & \multirow{6}{*}{87.668} \\
\hline & 29.8 & 0.897 & 0.7909 & 85.9201 & & & & \\
\hline & 6.094 & 1.151 & 0.8596 & 87.05953 & & & & \\
\hline & 4.864 & 1.18 & 0.9156 & 81.93206 & & & & \\
\hline & 8.219 & 1.1 & 0.8815 & 94.21422 & & & & \\
\hline & 23.04 & 0.9 & 0.8263 & 86.47135 & & & & \\
\hline
\end{tabular}
is in line with load - unload law. Show pushed the process more reliable.

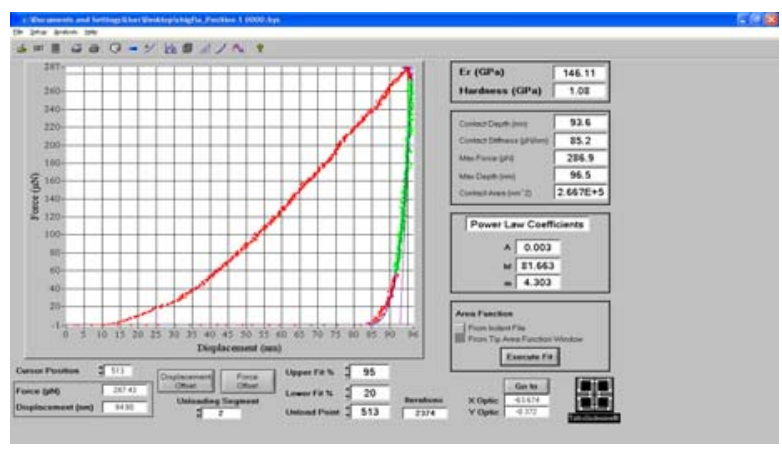

FIGURE II. ILLUSTRATES THE INDENTATION CURVES

TABLE I. A GROUP OF INDENTATION DATA.

\section{ORTHOGONAL DATA APPROXIMATION AND OPTIMIZATION}

During orthogonal optimization, load curve and select analog instrumentation indentation load curve of the same deep pressure 10 points $\left(0.5\right.$ to $3^{\mu m}$, interval $0.5^{\mu m}$ ) corresponding to the load deviation $\Delta \mathrm{i}$ and $\Sigma \Delta \mathrm{i}$ to optimize the target amount. Figure 3 shows the simulated load test load curve deviation between the curves. 
The experimental data obtained in the test group before adding 5, all relative optimization target amount $\Sigma \Delta$ corresponding sum is about to take a first level of factor $\mathrm{E}$ was 605.0805 , denoted I . The data from the second set of experiments 5 obtained by adding all the corresponding optimization target amount relative $\Sigma \Delta$ adding factor $\mathrm{E}$ is about to take 2 level was 566.6331, denoted II. The third set of numbers $11,12,13,14,15$ five experimental data obtained by adding 543.7966, denoted III. The fourth set of experimental data obtained by adding 518.7891, denoted IV. The fifth set of experimental data obtained by adding 498.5228, denoted V. Characterized by poor quantity of various factors on the extent of the impact test objectives, that is, 5 groups and subtracting the minimum and maximum recorded as R. The larger the poor $\mathrm{R}$ factors affecting its corresponding test target and the greater the amount of $\Sigma \Delta$ analyze orthogonal table can be found in important order for each factor is., The curve shown in Figure 4 after optimization.

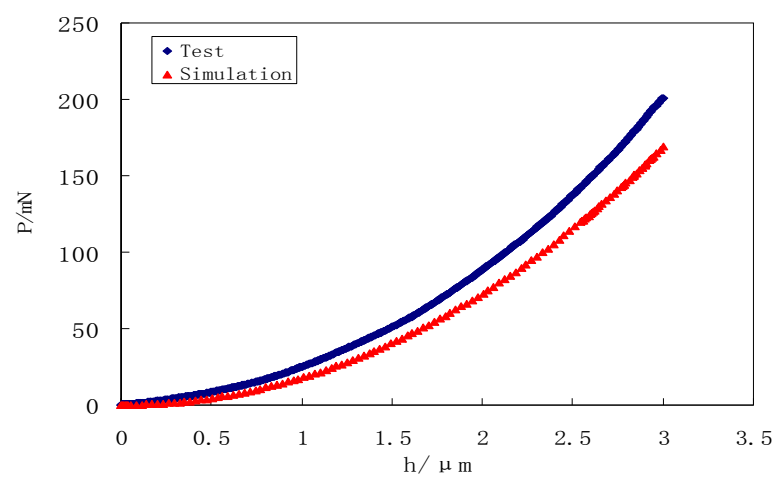

FIGURE III. THE SIMULATION OF LOAD DEVIATION BETWEEN THE CURVE AND THE TEST LOAD CURVE.

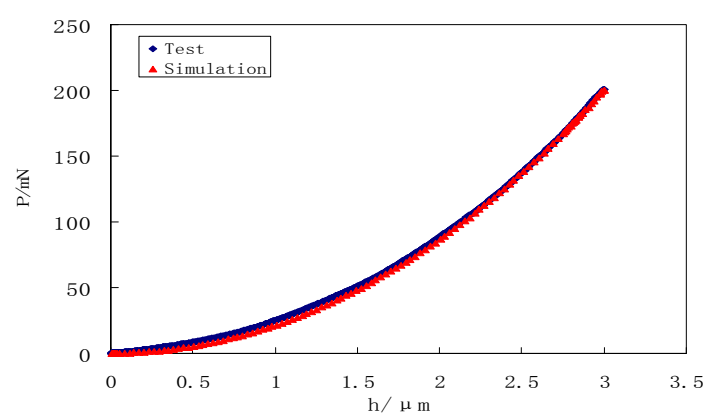

FIGURE IV. THE PARAMETRIC TEST LOAD CURVE OPTIMIZED LOADING CURVE WITH THE SAME ANALOG SCALES.

As shown, the effect is obvious, $\Sigma \Delta$ only $13.16 \mathrm{mN}$, you can find it both desirable than either a set of orthogonal test to prove the reliability of the orthogonal experiment also proved the feasibility of the proposed method.

\section{CONCLUSION}

By characterizing the constitutive relation of aluminum grating, nano-indentation experiments and numerical approximation research fitting method to obtain the following conclusions:
(1) Based on the analysis carved aluminum grating technology, the establishment of an aluminum grating dimensional characterizations approximation model;

(2) study the numerical approximation algorithm experimentally obtained by nano-indentation data, calculate the yield strength aluminum material property parameters, namely strain hardening exponent, and obtained more satisfactory aluminum material properties parameter elastic modulus and yield strength relationship by comparing the value of the deviation can be found in the obvious effectiveness of this approach, but also proved a reliable orthogonal experiments, provides a reference and basis for the characterization of the grating parameters characterize the relationship between aluminum and the constitutive model.

\section{REFERENCE}

[1] Fang T H, Jian SH R, Chu D S.Investigation of material properties of thin copper films[J].Applied Surface Science,2004,228(4):365.

[2] Ahn J H,Kwon D.Investigation of material properties through finite element modeling of microindentation test[J].Materials Science and Engineering A,2000,285(1):172.

[3] Erwin G. Loewen and Robert S. Wiley,Large diffraction grating ruling engine with nanometer digital control system,Proc. SPIE.[J].1987:88 95.

[4] Reed D T, Daully J W. A new method for measuring the strength and ductility of thin films [J]. J. Mater. Res., 1993, 8(7): 1542-1550.

[5] Shut C J, Cohen J B. Determination of yielding and debonding in $\mathrm{Al}-\mathrm{Cu}$ thin films from residual stress measurements via diffracrtion [J]. J. Mater. Res, 1991, 6 (5): 950- 956.

[6] Bader S. Comparison of mechanical properties and microstructure of $\mathrm{Al}$ (1wt, $\% \mathrm{Si})$ and $\mathrm{Al}(1 \mathrm{wt}, \% \mathrm{Si}, 0.5 \mathrm{wt}, \% \mathrm{Cu})$ thin films [J]. Thin Solid Films, 1995, 263(2): 175-181.

[7] SHANG Shuaihua, YANG Ping, LI Chun. Nano-indentation experiment of W/Al bilayer-film system and its finite element simulation[J]. ELECTRONIC COMPONENTS AND MATERIALS. 2009,28(11):60-63 